\title{
The Present Situation in Quantum Theory and its Merging with General Relativity
}

\author{
Andrei Khrennikov ${ }^{1,2}$ (D)
}

Received: 18 December 2016 / Accepted: 17 April 2017 / Published online: 3 May 2017

(C) The Author(s) 2017. This article is an open access publication

\begin{abstract}
We discuss the problems of quantum theory (QT) complicating its merging with general relativity (GR). QT is treated as a general theory of micro-phenomenaa bunch of models. Quantum mechanics (QM) and quantum field theory (QFT) are the most widely known (but, e.g., Bohmian mechanics is also a part of QT). The basic problems of QM and QFT are considered in interrelation. For QM, we stress its nonrelativistic character and the presence of spooky action at a distance. For QFT, we highlight the old problem of infinities. And this is the main point of the paper: it is meaningless to try to unify QFT so heavily suffering of infinities with GR. We also highlight difficulties of the QFT-treatment of entanglement. We compare the QFT and QM based measurement theories by presenting both theoretical and experimental viewpoints. Then we discuss two basic mathematical constraints of both QM and QFT, namely, the use of real (and, hence, complex) numbers and the Hilbert state space. We briefly present non-archimedean and non-hilbertian approaches to QT and their consequences. Finally, we claim that, in spite of the Bell theorem, it is still possible to treat quantum phenomena on the basis of a classical-like causal theory. We present a random field model generating the QM and QFT formalisms. This emergence viewpoint can serve as the basis for unification of novel QT (may be totally different from presently powerful QM and QFT) and GR. (It may happen that the latter would also be revolutionary modified.)
\end{abstract}

$\bowtie$ Andrei Khrennikov

andrei.khrennikov@1nu.se

1 International Center for Mathematical Modelling in Physics and Cognitive Sciences, Linnaeus University, Växjö, Sweden

2 National Research University of Information Technologies Mechanics and Optics (ITMO), St. Petersburg, Russia 
Keywords Quantum theory · Quantum mechanics · Quantum field theory · Quantum gravity - Infinities - Measurement theory · Non-hilbertian state space formalism . Non-real numbers in physics $\cdot$ Emergent theory

\section{Introduction}

The last year a few experimental groups performed the loophole free tests of violation of the Bell inequality [1,2], see [3-5]. This is really an exciting event in the quantum foundations! Leading experts in quantum foundations consider these tests as the final point in the exciting debate about the EPR-experiment, see, e.g., [6], [7] (cf., however, [8], [9]). In this euphoric situation the only commonly recognizable problem of the quantum community is the problem of merging QT (in any its form) with GR. The conventional viewpoint is that there is only one black cloud on the blue quantum sky: the evident impossibility to unify QT with GR. ${ }^{1}$ Does this picture reflect correctly the present situation in QT?

In this paper we shall analyze the fundamental problems of modern QT. We consider some of them as the main barrier preventing unification of QT and GR. ${ }^{2} \mathrm{We}$ point out that, in fact, two basic QT-models, QM and QFT, have some degree of independence-in general "they live their own lives". It is commonly stated, see, e.g., citations in appendix, that QFT is the fundamental theory and QM is its non-relativistic approximation. To be consistent with this viewpoint, we have to reduce the value of $\mathrm{QM}$ and treat it as a technical tool useful for concrete calculations in the non-relativistic limit. However, this is really not the case.

The indirect sign of the impossibility of diminishing the role of QM to the role of a non-relativistic computational tool is that the most exciting modern foundational discussions, especially those related to the recent quantum information revolution, are mainly going in the QM-framework. ${ }^{3}$

To complete this discussion on the role played by research on QM-foundations in general, I remark that it is quite common for experimentalists to claim that modern QM-foundational debates are of the minor value for real physics: QFT (which is claimed to be the real physical theory) is free of all QM-ambiguities- the relicts of the first period of development of QT. Surprisingly, this viewpoint is shared even by some experimentalists doing experiments related to quantum foundations. They like "cream on the top of foundational cappuccino" (as the Bell inequality), but at the same time they do not want to drink the rest: collapse of the wave function, the Born rule, the measurement problem and measurement theory (POVMs, theory of open quantum systems). Of course, this attitude is surprising for a theoretician. However, an experimentalist may really think that all these modern studies about the Born rule

\footnotetext{
1 This viewpoint was presented in numerous discussions of the author with experts in QT. Of course, they know well about other problems of QT. But all such problems are treated as technicalities which can be solved in future, see also appendix.

2 There has been some valuable work done in this direction, see, in particular, [10].

3 Only recently quantum information was coupled with QFT-foundations [11-15]. In general foundational QFT-studies are not so widely present at the quantum arena (see, however, e.g., [16-19]), see also appendix: $\mathrm{QM}$ versus $\mathrm{QFT}$ foundations.
} 
(e.g., where did it come from?) or quantum instruments are useless. There are detectors and there are clicks in them. Then the task of an experimentalist is to count the numbers of clicks in different detectors (and to control the level of noise, loopholes and other "technicalities"). We remark that this position matches well with the Copenhagen interpretation of QM and the views of N. Bohr: QM is about prediction of outcomes of classical measurement devices. ${ }^{4}$ This attitude of experimentalists to neglect the achievements of modern theory of quantum measurements is interesting to analyze and we shall discuss it in more detail in Sect. 3.

Personally I think that one cannot proceed with analysis of experiments without a theory of quantum measurements. And the latter was consistently developed (at the mathematical level of rigorousness) mainly in the QM-framework, namely, as theory of POVMs-based quantum instruments [20-22], see also [23] on discussion about interpretational issues. ${ }^{5}$ Typically it is difficult to handle the concrete measurement in the rigorous QFT-framework; often one has to refer to QM which is a rough approximation of QFT having many bad features. As the consequence, the most intriguing problem of modern quantum foundations, the problem of interrelation of local realism and QT, was treated (with a very few exceptions) only in the QM-formalism. In short, QFT cannot function successfully without QM and vice versa. We remark that QM needs QFT to secure that QT does not contradict special relativity, that nonrelativistic character of QM is just the consequence of the use of a convenient approximation, that, at least in principle, one can always refer to the existence of the explicit relativistic theory, QFT, see appendix for further discussion.

Now we come to the main theoretical problem of QFT. (The absence of measurement theory is the problem of applicability and in principle there can be a gap between a theoretical and observational models. ${ }^{6}$ ) This is the problem of QFT-divergences. This problem is well known. However, nowadays it is hidden "under the table": development of advanced mathematical techniques of renormalization made the impression that QFT is a consistent theory, see, e.g., citations in appendix. Personally I also practically forgot about this problem and in debates I always referred to QFT as the harmonically designed relative of QM. I argued that the corresponding foundational problems have to be lifted from QM to QFT for the proper analysis.

Recently going back home from the exciting conference DICE 2017 (Castigliancello, Italy) organized by T. Elze, I met a young Israeli post-doc and we started the conversation about QT and its merge with GR. His head was free of the mess of technicalities feeling my head. His questions might be naive. However, the fresh and curious insight in some problems can be of the great value. His questions revoked my former thoughts about the problems of QT, both in the QM and QFT fashions; in particular,

\footnotetext{
4 In fact, Bohr's concept of measuring devices is more complex. What Bohr says is that the observable parts of measuring instruments can be described by classical physics, while they also have quantum parts by means of which they interact with quantum objects.

5 For the QFT-measurement theory, see, e.g., Schweber [24], Schwinger [25]; from the foundational viewpoint, the pioneer papers of Bohr and Rosenfeld [26], [27] are still very interesting.

6 Such ideology was advertised by creators of so called Bild conception of the physical methodology, Hertz, Boltzmann, Schrödinger (see, e.g., [28], [29]). Nowadays it is often present in joint handling of two levels of description of nature, ontic and epistemic, see Atmanspacher et al. [30-32] and Khrennikov [8], see also Jaeger $[23,33,35]$ for discussions on quantum treatment of realism.
} 
about the problem of QFT-infinities. Since his PhD was in gravity, his questions had the strong gravitational flavor. This discussion is the basis of the presentation in Sect. 2. It has the form of a dialog, questions of the naive, but fresh minded young scientist to well educated (especially in mathematical problems of QT) professor. Formally saying, we analyzed the basic problems of QM-QFT in connection with the problem of merging with GR.

After this discussion my expectations for creation of new QT which will be free of all aforementioned problems and which will be harmonically merged with (new) GR increased to the level of strong optimism. Young generation whose fresh mind would not accept mathematical and physical inconsistency of the existing theories will finally revolutionize the modern physics, cf. with citation of P. Dirac at the very end of this paper.

In principle, after Sect. 2 the reader can jump directly to concluding Sect. 7 (What is to be done?). Other sections are more technical and longly. Sect. 3 is about measurement theory in QM versus QFT; may be it can be interested for experts in quantum foundations and quantum measurement theory. Sect. 4 (Is Devil in HIlbert state space?) enlightens the possibility to resolve at least some problems of QT by leaving the Hilbert (von Neumannian [36]) state space and proceed with a pair of dual linear spaces (in the spirit of P. Dirac [37]). Sect. 5 (Is Devil in real numbres?) revokes the very long discussion about the role of numbers in physics (rational, $p$-adic, and nonstandard numbers). Sect. 6 (Einstein's dream), see also [38], reminds about Einstein's dreaming for the classical field unification of all physical theories [39]. This Einsteinian way of thinking is illustrated by one special model, so called prequantum classical statistical field theory (PCSFT) [40].

\section{Problems of QT and its Unification with General Relativity}

As was already mentioned, recently going back home from the conference DICE 2017 I was staying at the platform with one young postdoc who asked me a variety of questions about QT (call this young scientist Y). We discussed education in physics in Israel ( $\mathrm{Y}$ is from this country). And I was positively surprised that his teacher (who was definitely from Russia) used the books of Landau and Lifshitz. This was the good point to continue conversation. Since I told him that I was interested in the mathematical foundations of QFT, he suddenly asked me whether it is really true that Feynman diagrams are divergent. I confirmed that QFT correlations should be regularized and started to give him examples of regularization procedures. For me, this was the mathematical routine. Therefore I was surprised by seeing dissatisfaction in his eyes. This was the fresh reaction of the brain which has not yet been structured by lecturing about the mathematical foundations of QFT. First of all Y was not happy at all that there is a variety of regularization procedures. He wanted just one and that it would be justified by laws of physics and not mathematics. He neither shared my enthusiasm on the possibility to get the right physical answers from complicated mathematical regularization procedures. The handmade nature of QFT regularizations was really disturbing for him. 
Then it happened that Y has already spent some time by thinking about infinities in QFT. He just was not sure that the state of art is so bad, that already so nicely sounded expression "Feynman diagram" is just the symbol covering the mess of regularization procedures of QFT. We continued our discussion at the stattion in Livorno, we still had $15 \mathrm{~min}$. For him, there are no infinities in the physical world and infinities are just the fruits of the human mind. I agreed with him completely. Then he made the point: "I think that the appearance of infinity is just a sign that some theory is applied outside its real domain of application". And this statement generated a chain of my recollections.

"Late Dirac" was neither satisfied by modern QFT and also because the presence of infinities. ${ }^{7}$ This dissatisfaction was especially strongly expressed in Dirac's book [42] in which Dirac asked for creation of new QFT which would be free of divergences. Y was curious whether Dirac gave some insights towards creation of new divergences free QFT. My recollection was that Dirac was sure that the main problem of modern QFT is in very formal treatment of the vacuum state $|0\rangle$. The real physical vacuum has a complex structure and this structure cannot be reduced to operation with the symbol $|0\rangle$.

The next question was again surprising for me: "How could it happen that scientists working in QFT (and more generally QT) did not put all their efforts to create a new divergence free QFT?" It seems that the answer was already in his own remark that infinities are well accommodated in the human brain. People did not treat the appearance of infinities as a sign of inapplicability of a theory. I pointed to the Big Bang theory with its initial singularity which is also well accommodated in our brains, but, of course, has nothing to do with physics. The Bing Bang initial singularity should be a powerful stimulus to create a new theory, e.g., in the spirit of the recent studies of V. G. Gurzadyan and R.Penrose [43]. However, this did not happen and we peacefully live starting with the singular Big Bang.

Then Y told: "Regarding Dirac's attempt to reorganize QFT by clarifying the role of vacuum, was it not just a step towards quantum gravity?" Yes, it can be seen in this way.

And it became clear for us both that we should not try to unify QT suffering of divergences (at the QFT level) with GR, that first we have to resolve the biggest, but commonly ignored (nowadays) problem of QT, namely, creation of QFT without infinities and their handmade regularizations. My next recollection was that infinities are not present in nonlocal versions of QFT, see e.g. [44]. Thus there is some coupling between suffering from infinities and enjoying locality. I told that by introducing the elementary length $L$ and making impossible to go beyond it we can proceed without infinities. My friend was completely fine to live in the space which is not infinitely divisible and he preferred such world to the QFT-world with infinities.

Then Y made the interesting point: "See, we all know from Bell's theorem and its experimental verification that QM is nonlocal! Why do people have such a problem with invention of nonlocality to QFT? It is clearly nonlocal by Bell's theorem!?” And here I started to present my favorite story about nonlocality of QM versus locality of QFT:

\footnotetext{
7 In the early 1980s, P. Dirac told E. Witten that the most important challenge in physics was to get rid of infinity [41].
} 
QM is an approximation of QFT. Do you agree? (“yes!”) The real QT is QFT and not QM at all. ("yes!") In particular, QM is nonrelativistic theory. ("yes!") There is a theory which is called "relativistic QM", but it suffers of such problems that it is better to be nonrelativistic (Sect. 3). Therefore if one were defining "quantum nonlocality" simply as the absence of relativistic invaraince, then the statement about "nonlocality of QM" would be trivial. It is the good place to remark that locality of QFT is defined precisely in the sense of relativistic invariance. What is about nonlocality of QM? This is a very special notion which surprisingly does not refer to space-time at all. In particular, there is no space-time in the formulation of Bell's theorem. ("yes!") Nonlocality of QM is based on action at a distance which existence is seen as a consequence of Bell's theorem and its experimental verification. ${ }^{8}$ Thus the notion of locality in QM and QFT have very different meanings. ("uf!") Nowadays we are excited by having the QM-nonlocality, but at the same time we are afraid of nonlocal versions of QFT.

$\mathrm{Y}$ has a very good sense of logic and he was curious why nobody tried to proceed another way around and formulate the principle of action at a distance in the QFT framework? Up to my knowledge such attempts have been done, but my impression was that one cannot proceed consistently. QFT with action at a distance seems to be as bad as QM with relativity. In particular, I recalled that even the notion of entanglement in QFT is not free of problems. "Why?"

One of the theoretical problems of the QFT-treatment of the Bell-type argument is that entanglement depends on the frame.

Of course, this is also not such a surprise, since entanglement (per se) is a mathematical definition, i.e., a physical system can be separable or entangled with respect to a chosen factorization of the total Hilbert space which describes the quantum states. Indeed, the choice of factorization allows for pure states to switch unitary between separability and entanglement. However, usually the experimental setup fixes the factorization and applying local unitary transformations does not change the entanglement properties. But, as was already stressed, measurement is properly described only in the quantum mechanical and QFT framework. My impression is that the main problem behind the QFT-treatment of action at a distance is the absence of measurement theory corresponding to QFT! Y: "Well, I suspected this!"

I finished my story by pointing that even among top experts there is not consistency in views on measurement theory in the QFT framework. I had a few conversations with top experimentalists in quantum optics who are also well known in quantum foundations and they agreed that QFT has no measurement theory and this is one of the problems of modern QT. At the same time other top experimentalists did not see any specialty of QFT treatment of measurements comparing with QM (see Sect. 3 for further discussion).

\section{Measurement Theory: QM Versus QFT}

QM-based measurement theory started from the Born rule, then its preliminary formalization was performed by von Neumann and it was based on the projection postulate,

\footnotetext{
8 It should be pointed out that this is a different sort of "action" from that which concerned, for example, Newton: as it cannot be used for signaling.
} 
finally it was formulated as theory of POVM-representable measurements (more generally theory of quantum instruments) in connection with theory of open quantum systems, see [20-23] for detailed presentation of the present state of art. Theory of quantum instruments is mathematically rigorous and clear theory, straightforwardly applicable to the basic quantum experiments.

It is important to remark that the only mathematically and conceptually sound QM is nonrelativistic QM. One may disagree and point to relativistic QM. However, the latter suffers of a number of difficult problems, e.g., the problem of relativistic localization (position). By Hegerfeldt's theorem: Einstein causality implies that there can be no spectral measure solution to the localization problem in relativistic QM.

Thus, for a moment, we have the well developed theory of nonrelativistic quantum measurements. Relativistic treatment is presented by QFT-based measurement theory, e.g., Schweber [24], Schwinger [25]. My personal opinion (confirmed by conversations with a few top experts in quantum measurement theory) is that the QFT-measurement theory is far from the level of mathematical rigorousness and clearness approached by the QM-measurement theory. But this is just the private opinion, cf. with coming analysis of the viewpoints of experimentalists.

I shall present a "typical viewpoint" representing very general attitude of experimentalists towards quantum measurement theory, a kind of general synthetic statement extracted from conversations during the Växjö series (2000-2016) of conferences on quantum foundations:

One may say that an experimentalist does not need quantum measurement theory: neither in the original von Neumann form (Hermitian operators and the projection postulate) nor in the modern form (POVMs and quantum operations). An experimentalist needs only probabilities for detection of events. These probabilities are provided by $Q F T$.

In contrast to QFT, QM is only applicable for the Minkowski gauge. (It works only for the flat space.) Moreover, the measurement theory in the QFT framework is, in fact, more simple and fundamental than the QM measurement theory (von Neumann's theory or modern theory of quantum instruments). Here, in QFT, one can proceed following the ideas of Einstein (see, e.g., Einstein and Infeld [39]):

1. Only fields do exist.

2. Any interaction of fields comes with an exchange of their excitations, "particles".

3. Any measurement can be treated as the interaction on the observed field with the field of the measurement apparatus (may be even including electromagnetic field in experimentalist's brain).

4. Any measurement then merely consists of counting the quantum particles arising from the interaction involving the two fields: measured input field and measurement apparatus field.

QFT express this counting procedure by the measurement and calculation of the current $J$, detected by the amount of 'clicks' of the counting device. This measurement of $J$ by counting clicks is exactly what in being measured every day at the LHC machine in Geneva. 
Do you find in this simple scenario any need for POVM, or 'entanglements' a la Zurek, or quantum instruments a la Davies and Ozawa, or other theoretical nasty procedures?

It is clear that this viewpoint has some basis behind it. It has to be analyzed in more detail than it is possible in this paper (which is not devoted to quantum measurement theory). For me, this position of experimenters is very close to Bohr's position: QT is about predictions of outputs of classical measurement devices. The first part of the "experimentalist's statement" about measurement theory would be supported by the majority of experimentalists. The second part related to the field picture of measurement process represents the more specific viewpoint on the quantum measurement theory which is characteristic for some part of the experimental community. From this viewpoint both "systems" and measurement devices are represented by fields. The above reference to Einstein makes the impression that these fields are treated classically. However, this may be not the case. In general my impression is that often experimentalists treat the (operator-valued) fields of QFT in the same manner as classical fields.

We remark that "Einstein never accepted the QFT theory, because it shared all the 'problems of QM', or what he so perceived, most especially its fundamentally nonrealist and acausal, and thus statistical nature. In fact it is not clear to what degree he grasped or, given this character of the theory, even wanted to grasp QFT, or QED. At one point, he developed an interest in Diracs equation, as a spinor equation, and he used it, in his collaborations with W. Mayer, as part of his program for the unified field theory, but, again, conceived as a classical-like field theory, modeled on general relativity, and in opposition to quantum mechanics and, by then, quantum field theory. Accordingly, he only considered a classical-like spinor form of Diracs equation, thus depriving it of (Einstein might have thought 'freeing' it from) its quantum features, most fundamentally, discreteness ( $h$ did not figure in Einsteins form of Diracs equation), and probability. Einstein hoped, but failed to derive discreteness from the underlying field-continuity. Einstein was primarily interested in the mathematics of spinors, which he generalized in what he called semi-vectors" (private communication with A. Plotnitsky).

One may say that experimentalists still keep to the orthodox Copenhagen interpretation, because they consider the present measurement theory as unsatisfactory.

However, I disagree that transition from QM to QFT may simplify measurement's picture. I would like to point to the following complication due to the QFT-treatment, see also Plotnitsky [14], Chap. 6. Since QM is a nonrelativistic model, the space-time can be, in principle, excluded from consideration. The ontological interpretation of the wave function $\psi=\psi(t, x)$ is, although possible, not so much supported by the recent development of quantum foundations. We can (by following Schrödinger) to interpret a quantum state as an expectations catalog. The Schrödniger dynamics, $\psi_{t}=U_{t} \psi_{0}$, need not be coupled to processes in physical space-time.

We remark that von Neumann measurement theory does not describe the process of interaction of a quantum system and a measurement device in physical space-time. However, by the above argument this is fine (in the nonrelativistic QM-framework). In QFT, physical space-time reappeared in whole its power, very similarly to classical field theory. Quantum fields are functions on the space-time, the "only difference" 
from classical field theory is that quantum fields are operator valued. The proper QFTmeasurement theory has to take the space-time structure into account and to present the space-time based picture of interactions of quantum systems (fields) with measurement devices (represented by quantum or even classical fields). ${ }^{9}$ To my knowledge, there is no such a theory.

It seems that by speaking about "simply counting procedures" we shadow thremedous difficulties of the theoretical description of these procedures As Pais said years ago [45], p. 325, but this still true:

"Is there a theoretical framework for describing how particles are made and how they vanish? There is: quantum field theory. It is a language, a technique, for calculating the probabilities of creation, annihilation, scattering of all sorts of particles: photons, electrons, positrons, protons, mesons, others, by methods which to date invariably have the characters of successful approximations. No rigorous expression for the probability of any of the above-mentioned processes has ever been obtained. The same is true for the corrections, demanded by quantum field theory, for the positions of energy levels of bound-state systems [e.g., atoms]. There is still a [Schrdinger] equation for the hydrogen atom, but it is no longer exactly soluble in quantum field theory." (Pais speaks of particles, but a field interpretation does not change anything in this respect.)

\section{Is Devil in Hilbert State Space?}

If one reads the works P. Dirac carefully, starting with his famous book [42] and paying especial attention to his book devoted to QFT [46], it becomes clear that P. Dirac suspected that the fundamental problems of QT, especially divergences in QFT, are consequences of the use of the Hilbert space as the mathematical model of the state space. In fact, he started his way to construction of the mathematical formalism of QM not with the Hilbert state space (in contrast to von Neumann [36]), but with a pair of two dual linear spaces. Denote them $E_{+}$and $E_{-}$. The elements of one of them are called ket-vectors and of another bra-vectors; the former are denoted as $|\psi\rangle$ and the latter as $\langle f|$. The duality form for these two spaces is denoted as $\langle f \mid \psi\rangle$. Only later in the text of his book [42] Dirac identified these spaces $E_{+}=E_{-}=H$ and $H$ can be mathematically treated as the Hilbert space. Of course, such identification simplified the mathematical formalism. The main physical argument in favor of such identification is that only in Hilbert space we can proceed with the Born rule providing the probabilistic interpretation of QM. We shall discuss this statement later.

Now we point out that, in spite of Hilbertization of his formalism, P. Dirac continued to operated with two types of vectors. The latter seems to be totally meaningless, cf. with the book of von Neumann [36]. However, careful analysis shows that (as from the very beginning) P. Dirac used the ket and bra vectors as the elements of dual vector spaces. Thus elements of $E_{-}$can be treated as linear functionals on the vector space $E_{+}$. (These functionals are, in fact, the basic blocks to determine observables.) The

\footnotetext{
9 The problem cannot be disregarded by formal transition to the particle representation in the Fock space. Creation and annihilation operators are still functions defined on physical space-time.
} 
space of ket-vectors is really the state space. However, the space of bra-vectors $E_{-}$is, in fact, the space of linear functionals on the state space $E_{+}$.

In this scheme an observable $A$ is determined by a sequence of functionals, bravectors, $A=\left(f_{1}, \ldots, f_{n}, \ldots\right)$. Each functional represents measurement of some value $a_{i}$ of the observable $A$. For mathematical simplicity, we consider observables with discrete ranges of values; we also suppose different functionals represent different values (given by real numbers).

Then the Born rule has the following form. For systems prepared in the state $\psi \in E_{+}$ and the observable $A=\left(f_{1}, \ldots, f_{n}, \ldots\right), f_{j} \in E_{-}$, the probability to observe the outcome $a_{i}$ is given by the rule:

$$
p\left(A=a_{i} \mid \psi\right)=\frac{\left|\left\langle f_{i} \mid \psi\right\rangle\right|^{2}}{\sum_{j}\left|\left\langle f_{j} \mid \psi\right\rangle\right|^{2}} .
$$

If $\sum_{j}\left\langle f_{j} \mid \psi\right\rangle^{2}=\infty$, then we say that the observable $A$ cannot be measured for this state. The latter does not mean that it is something wrong with the state $\psi$, that it is unphysical. It can be that a variety of other observables are measurable for $\psi$. For some $B=\left(g_{1}, \ldots, g_{n}, \ldots\right), \sum_{j}\left\langle g_{j} \mid \psi\right\rangle^{2}<\infty$. This is a natural physical situation; two procedures, preparation and measurement, should be consistent.

In the modern functional analysis, the space of ket-vectors (the state space) $E_{+}$ can be treated as a topological linear space, a linear space endowed with some topology such that the operations of addition of vectors and multiplication of scalars are continuous. The space of bra-vectors (elementary components of observables) $E_{-}$is represented as the dual space, the space of continuous linear functionals on $E_{+}$. For example, $E_{+}$can be chosen as the space of Schwartz test functions $E_{+}=\mathcal{S}\left(\mathbf{R}^{3}\right)$ and its dual space is the space of Schwartz distributions $E_{-}=\mathcal{S}^{\prime}\left(\mathbf{R}^{3}\right)$.

To make closer analogy with the Hilbert space case, consider a topological linear space $E_{+}$having a (topological) basis, i.e., a system of vectors $\left(e_{1}, \ldots, e_{n}, \ldots\right)$ such that any vector $\psi$ can be expanded uniquely with respect to this system

$$
\psi=\sum_{j} x_{j} e_{j}, x_{j} \in \mathbf{C},
$$

and the series converges in the topology of $E_{+}$. Consider the system of linearfunctionals on $E_{+}:\left(f_{1}, \ldots, f_{n}, \ldots\right),\left\langle f_{j} \mid \psi\right\rangle=x_{j}$, i.e., $\left\langle f_{j} \mid e_{i}\right\rangle=\delta_{j i}$. Then this system of functionals is a basis in the dual space $E_{-}$. The latter is endowed with so called weak topology. ${ }^{10}$ The systems of vectors $\left(e_{1}, \ldots, e_{n}, \ldots\right)$ and functionals $\left(f_{1}, \ldots, f_{n}, \ldots\right)$ are biorthogonal bases. The expansion (2) can be written as

$$
\psi=\sum_{j}\left\langle f_{j} \mid \psi\right\rangle e_{j} .
$$

Now we remark that we could proceed another way around: to start with a topological basis in the dual space $E_{-},\left(f_{1}, \ldots, f_{n}, \ldots\right)$, and construct its biorthogonal basis in $E_{+}$.

\footnotetext{
10 To make everything consistent, we have to consider the special class of topological linear spaces, so called locally convex spaces. In such spaces each point has the basis of convex neighborhoods.
} 
The weak topology on $E_{-}$has the property that the dual space for $E_{-}$coincides with $E_{+}$.

Now consider the observable $A$ given by some basis $A=\left(f_{1}, \ldots, f_{n}, \ldots\right)$. Let $\left(e_{1}, \ldots, e_{n}, \ldots\right)$ denote the corresponding basis in the state space. Then this observable can be represented by the linear operator $\hat{A}: E_{+} \rightarrow E_{+}$, acting as $f \rightarrow$ $\sum_{j} a_{j}\left\langle f_{j} \mid \psi\right\rangle e_{j}$. In the Dirac notation it can be written as

$$
\hat{A}=\sum_{j} a_{j}\left|e_{i}\right\rangle\left\langle f_{j}\right| .
$$

In Hilbert space we can identify the vector and functional counterparts of the system of the biorthogonal bases, set $f_{j}=e_{j}$ and obtain the standard spectral decomposition of a Hermitian operator.

$$
\hat{A}=\sum_{j} a_{j}\left|e_{i}\right\rangle\left\langle e_{j}\right| .
$$

Consider now a rigged Hilbert space based on the dual pair $E_{+}, E_{-}$:

$$
E_{+} \subset H \subset E_{-}
$$

where $H$ is a Hilbert space and the injections of $E_{+}$into $H$ and $H$ into $E_{-}$are continuous with respect to the topologies on these spaces. ${ }^{11}$ One of common examples is based on the Schwartz spaces and the $L_{2}$-space:

$$
\mathcal{S}\left(\mathbf{R}^{3}\right) \subset L_{2}\left(\mathbf{R}^{3}\right) \subset \mathcal{S}^{\prime}\left(\mathbf{R}^{3}\right)
$$

By considering Hermitian operators in the Hilbert space $H$ we reduce essentially the class of possible observables. The main point of Dirac was that by considering quantum dynamical equations in $H$ (for observables, i.e., in the Heisenberg picture) we restrict essentially the class of possible dynamics. Infinities are induced by our attempt to represent the $E_{-}$-dynamics as the $H$-dynamics.

In the Hilbert space model the Heisenberg and Schrödinger pictures are equivalent. In the general dual space model, the situation is more complicated. We can repeat the above scheme by selecting $E_{-}$as representing the state space and $E_{+}$as representing observables. In the case of $E_{+}=\mathcal{S}\left(\mathbf{R}^{3}\right)$ and $E_{-}=\mathcal{S}^{\prime}\left(\mathbf{R}^{3}\right)$ this state-observable inversion leads to huge space of states and small space of observables. Now a state $\psi$ is represented by an arbitrary vector $\psi \in E_{-}$and an observable is represented as $A=\left(f_{1}, \ldots, f_{n}, \ldots\right), f_{j} \in E_{+}$. The Born rule has the form:

$$
p\left(A=a_{i} \mid \psi\right)=\frac{\left|\left\langle\psi \mid f_{i}\right\rangle\right|^{2}}{\sum_{j}\left|\left\langle\psi \mid f_{i}\right\rangle\right|^{2}} .
$$

\footnotetext{
11 To have a mathematically rich model, the topological space $E_{+}$should belong to the class of so called nuclear spaces; all basic spaces of functional analysis are of such a type.
} 
Consider again a rigged Hilbert space, see (9). Then, from Dirac's viewpoint, the main problem of QT is in attempting to restrict the state space of a quantum system to the fixed Hilbert space $H$. In fact, the real state dynamics takes place not in $H$, but in $E_{-}$. In the example (7), states are accommodated in the space of distributions and not in the $L_{2}$-space.

The selection of a Hilbert space $H$ corresponds to the selection of a fixed vacuum. Dirac pointed out that the real state dynamics' need not be concentrated in the space corresponding to this concrete vacuum (which choice corresponds to our laboratory conditions); for infinitely small instance of time the state can be kicked out this fixed Hilbert space $H=H_{0}$.

If one wants to treat a quantum system as living in a Hilbert state space, then time-dependent rigged Hilbert spaces have to be explored:

$$
E_{+} \subset H_{t} \subset E_{-}
$$

Now we remark that the example (7) is only of the illustrative value, since it represents Dirac's ideology in the QM-framework, i.e., for systems with the finite number of degrees of freedom. To explore this scheme for QFT, one has to consider spaces of test functions and distributions on infinite-dimensional spaces. Non-Hilbertian approach to quantization of systems with infinite number of degrees of freedom was established by O.G. Smolyanov et al. [47-51].

In particular, an attempt to realize Dirac's idea about state-dynamics which is instantaneously kicked of the Hilbert state space (the Fock space) at the mathematical level was presented in [52]. But there is still the huge gap between mathematical modeling and real physics.

In this paper we consider constraints making difficult unification of QT and GR. The use of the Hilbert state space is one of such constraints, see, e.g., 't Hooft [53]: "The standard Hilbert space procedure for quantum mechanics does not go well with gravitation, curved space time and cosmology." At the same time theory of distributions is well adapted to manifolds. This section is aimed to remind about this important constraint. At the same time we are well aware that Dirac's own attempts to resolve quantum foundational problems by proceeding in the non-Hilbertian framework were not successful.

\section{Is Devil in Real Numbers?}

We remark that the real numbers appeared in physics as a mathematical supplement of the use of Newtonian differential and integral calculus. ${ }^{12}$ The use of real numbers in physics was the culmination of long struggle between supporters of the "continuous and discrete viewpoints" on modeling of nature. The continuous model is rooted to the works of Aristotle. In the modern language his ideas can be formulated as follows: continuous geometry for the physical world and discrete geometry for the

\footnotetext{
12 If physicists would follow Leibniz, then the modern physics were based on some version of nonstandard analysis [69].
} 
mental world. Democritus claimed that the world is build of atoms, indistinguishable elementary blocks of nature. ${ }^{13}$

Newton's idea about infinitely divisible continuum was later elaborated mathematically and in 19th century the rigorous mathematical model of the field of real numbers was created. This model worked perfectly in classical mechanics and it was successfully extended to cover field theory. We remark that even theory of electricity was based on a kind of continuous electric fluid. Discoveries of electron and atom recovered Democritus discrete picture of nature. Then M. Planck showed that the problem of black body radiation can be solved if the energy exchange between matter and radiation is modeled by using discrete portions of energy, quanta. (We remark that already Boltzmann used in mathematical calculation portioning of energy by discrete "quanta" $\epsilon$. Thus M. Planck proceeded in this direction by assigning to $\epsilon$ the concrete value $\epsilon_{v}=h \nu$.) Einstein made the next step and claimed that energy of the radiation is quantized not only in the process of the energy exchange between matter and field, but the electromagnetic field by itself is quantized, in vacuum. We also point to Bohr's model of atom in which electrons could not move freely in the real space, but only choose the special discrete set of orbits. This was the right time to look for a new mathematical model representing these discrete features of micro-world. However, the real numbers were peacefully incorporated in the mathematical formalism of QM. From this viewpoint, QM is an attempt to model discrete nature with the aid of infinitely divisible continuum.

It might be that some strange features of QM and QFT, including divergences in the latter, are just the mathematical artifacts of using the real numbers model. This viewpoint is not new and its different versions were presented by many scientists, see the monograph [54] for a discussion and references about this topic. One class of proposals is based on the use of finite number fields, instead of the field of real numbers. Another class is based on the idea that only rational numbers can be treated as "physical numbers". Starting with the field of rational numbers $\mathbf{Q}$, one can try to construct new number fields. ${ }^{14}$ Surprisingly mathematics gives us only two possibilities to proceed in this way: either to the field of real numbers $\mathbf{R}$ or to the fields of $p$-adic numbers $\mathbf{Q}_{p}$, where $p>1$ is a prime number determining the field, see, e.g., [54]. The latter combines the discrete and continuous features. $P$-adic analysis can be considered as the discrete analog of Newton's analysis for continuous entities. Mathematically $\mathbf{Q}_{p}$ is constructed in the same way as $\mathbf{R}$, as completion of $\mathbf{Q}$, but with respect to the special $p$-adic metric. This metric is so called ultrametric; not only the standard triangle inequality, but even strong triangle inequality holds. By the latter in each triangle the length of third side is always majorated by the maximum of the lengths of two other sides. This feature of metric on $\mathbf{Q}_{p}$ induces unusual features of $p$-adic geometry.

Starting with the pioneer paper of I. Volovich [55] in 1987 (see also [56], [57]), $p$-adic theoretical physics has been rapidly developing and covering all basic areas of physics, but with the emphasize on string theory, see, e.g., the monographs [58], [59], [54] and the recent review [60]. String theory models nature at the fantastically

\footnotetext{
13 Plato hated Democritus' idea and asked to burn Democritus' writings. We remark that, although Newton's calculi were based on infinitely divisible continuum, his model of light was of corpuscular nature.

14 Algebraic structures for which all basic arithmetic operations, addition, subtraction, multiplication, and division, are well defined.
} 
small space and time intervals, at the Planck scale. It was natural to question the Newtonian model of space-time based on the field of real numbers R. In particular, one questioned infinite divisibility of space-time intervals, the determining feature of the real continuum. Therefore the $p$-adic space-time was welcome as one of possible candidate for the mathematical model of the Planck space-time. The international team of researchers, Volovich, Vladimirov, Aref'eva, Witten, Dragovich, Frampton, did a lot in this direction, see [60]. However, since string theory by itself has no direct relation to real physics, the $p$-adic string theory cannot be used to justify the use of the fields of $p$-adic numbers $\mathbf{Q}_{p}$ in physics, in particular, in QFT.

We remark that the $p$-adic theoretical physics explores two mathematical models. In both models the space is $p$-adic, i.e., instead of $\mathbf{R}^{n}$, one uses $\mathbf{Q}_{p}^{n}$.

In most developed model [58], one uses the functions $\phi: \mathbf{Q}_{p}^{n} \rightarrow \mathbf{C}$, where $\mathbf{C}$ is the ordinary field of complex numbers. For example, in corresponding $p$-adic QM wave functions are complex valued, as in the standard QM. The state space is the space of square integrable functions (with respect to the Haar measure on $\mathbf{Q}_{p}^{n}$ ), $H=$ $L_{2}\left(\mathbf{Q}_{p}^{n}\right)$, and this is the usual complex Hilbert space. Spectra of operators representing observables are subsets of $\mathbf{R}$. The standard probability interpretation, the Born rule, can be used. The main difference from standard QM is that in $p$-adic QM it is impossible to construct an analog of operators of position and momentum, a kind of Heisenberg algebra. ${ }^{15}$ Mathematically the $p$-adic model with $\mathbf{C}$-valued functions can be treated as a part of harmonic analysis on locally compact groups (the fields of $p$-adic numbers are locally compact additive groups). ${ }^{16}$

In another model of $p$-adic theoretical physics (see [59], [54], [61-64]), both variables and values are $p$-adic. ${ }^{17}$ This model is farer from standard QM than the $\left[Q_{p}^{n} \rightarrow \mathbf{C}\right]$-model. The main mathematical problem of the "genuine $p$-adic model" is that the analog of the Born rule leads to $p$-adic quantities having the meaning of probabilities. This is the good place to remark that real numbers infiltrated into physics much deeper, not only space-time coordinates, but even probabilities are represented by real numbers. In a series of works of the author (see, e.g., [59], [54], [65]), there was designed probability theory with $p$-adic valued probabilities. They are defined in the spirit of von Mises frequency theory of probability, as the limits of frequencies,

$$
P(a)=\lim _{N \rightarrow \infty} \frac{n_{a}}{N}
$$

where $n_{a}$ is the number of observations of the result $a$ and $N$ is the total number of observations. Here the limit is taken with respect to the $p$-adic metric. We remark

\footnotetext{
15 The standard Schrödinger representation of $\hat{x}_{j}$ and $\hat{p}_{j}$ based on the multiplication and differentiations cannot be extended to $p$-adic QM by the purely mathematical reason: it is impossible to multiply $p$-adic and real (complex) numbers. One cannot multiply a function $\phi: \mathbf{Q}_{p}^{n} \rightarrow \mathbf{C}$ by one of its variables $x_{j} \in \mathbf{Q}_{p}$. This also implies that it is impossible to define derivatives of such functions [59].

16 However, the presence of the field structure, i.e., combination of the operations of addition and multiplication, and special features of the distance on $\mathbf{Q}_{p}$ which is ultrametric make $p$-adic analysis essentially richer and it has some very special features which are not common for locally compact groups in general.

17 The latter can belong to quadratic and more general algebraic extensions of $\mathbf{Q}_{p}$, analogs of the field of complex numbers $\mathbf{C}$ which is the quadratic extension of $\mathbf{R}, \mathbf{C}=\mathbf{R}(\sqrt{-1})$.
} 
that frequencies always belong to the field of rational numbers $\mathbf{Q}$. This field can be embedded both in the fields real and $p$-adic numbers, $\mathbf{Q} \subset \mathbf{R}$ and $\mathbf{Q} \subset \mathbf{Q}_{p}$ for any $p>1$. Therefore any sequence of frequencies $\left\{q_{N}(a)=\frac{n_{a}}{N}\right\}$ can be handled both as a sequence in $\mathbf{R}$ and in $\mathbf{Q}_{p}$. $P$-adic probabilities are defined for sequences having the limits in $\mathbf{Q}_{p}$.

This theory questioned applicability of the standard law of large numbers. The existence of the limit (10) in $\mathbf{Q}_{p}$, typically implies nonexistence of such a limit in R. ${ }^{18}$ Thus reconsideration of QT on the basis the "genuine $p$-adic model" should lead to reconsideration of the role and interpretation of probability in QT. The first steps in this direction were done in author's works [59], [54], [66] (the latter paper is devoted to the $p$-adic probabilistic treatment of the EPR-experiment). However, these studies are still of purely theoretical nature.

We also point to the $p$-adic attempts to beat quantum divergences by considering $p$ adic quantities, instead of the real ones (see, e.g., the papers of Cianci and Khrennikov [67], [68]).

A very different program of reconsideration of QT through playing with the basic number field is based on the Leibniz approach to calculus. We recall that he interpreted infinitely small and large quantities on the same grounds as finite ones. In Leibniz's calculus one can operate with, e.g., infinitely large numbers, add them to finite numbers, subtract them, multiply, and divide. It is very attractive to apply the Leibniz approach to handle QFT infinities. One of the rigorous mathematical models for Leibniz's calculus is based on the field of nonstandard numbers (see, e.g., Robinson's book [69]). It is typically denoted by the symbol ${ }^{\star} \mathbf{R}$. It is an extension of $\mathbf{R}$ - to include infinitely small and large quantities. ${ }^{19}$ The applications of nonstandard analysis to QFT were considered by a few authors [70-73]. ${ }^{20}$

In short, development of $p$-adic physics (based on consideration of only rational numbers as physical numbers) can be characterized as very successful theoretically, but its models are still far from experimental verification. The same can be said about nonstandard physical models. Nevertheless, it is useful to keep in mind this way of

\footnotetext{
18 We remark that the standard "real law of large numbers" is, in fact, the basis of the modern scientific methodology: only observations satisfying it are considered as scientifically meaningful. Statistical data violating this law is considered as output of badly performed observations.

19 The $p$-adic program of reconsideration of QT (and physics in general) is based on the idea that a kind of the discrete model has to serve as the mathematical basis of such reconsideration. The nonstandard program is based on the idea that one should not afraid infinities at all, accept them as physical quantities. Thus the $p$-adic and nonstandard programs question the standard real physics, but from the opposite sides. On the other hand, we can mention the common feature of the $p$-adic and nonstandard fields. They both are non-Archimedean fields. The Archimedean axiom is violated. This axiom can be treated (due to Volovich [55]) as a measurement axiom. In the Archimedean measurement theory by having two quantities $l$ (e.g., unit of measurement) and $L$ it is always possible to measure $L$ (at least approximately) with the aid of $l$. In the non-Archimedean theory this natural feature of the measurement process can be violated.

20 I was lucky to cooperate closely with S. Albeverio who did a lot for establishing of the nonstandard approach of QFT, e.g., [72], [73]. In early 1990th he was very enthusiastic and his expectations for nonstandard reconsideration of QFT were really great. It seems that later he lost the interest to this program. (It may be that his later interest to the $p$-adic approach, see, e.g., [63], [64] was stimulated by disillusion in possibilities of the nonstandard approach.) In general nowadays the nonstandard analysis is practically dead, only a very few people still try to use it.
} 
reconsideration of QT (i.e., exclusion of real numbers from quantum physics and playing with other number fields and more general algebraic structures).

\section{Einstein's Dream}

By the Copenhagen interpretation of QM this theory is complete, i.e., it is impossible to find a finer description of physical phenomena in the micro-world than the wavefunction description. ${ }^{21}$ For the fathers of this interpretation, Bohr, Heisenberg, Pauli and Fock, completeness was the straightforward consequence of the existence of the fundamental quantum of action, given by the Planck constant $h$. They did not need to search for no-go theorems. The Heisenberg uncertainty relation was the deciding "no-go theorem". The completeness of QM implies that it is impossible to emerge it from some subquantum classical-like (causal) theory (see, e.g., Jaeger $[23,33,35]$ for detailed analysis).

Nowadays a variety of no-go statements prevents treatment of QM as emergent theory. Moreover, nowadays the really misleading element, quantum nonlocality, plays the crucial role in debates on the possibility to emerge QM from a classical-like model, $[1,2,7]$. It is widely claimed that such emergence is possible only by assuming the presence of nonlocal interactions of the form of the Bohm quantum potential. Bohmian-type emergence of QM is totally foreign to GR and the general Einstein attitude to reproduce QM/QFT from a (local) classical field model, see Einstein and Infeld [39]. Such emergence cannot serve to unification of QT and GR, since the latter is local theory.

However, by distancing from statements such as the Bell no-go theorem we can consider the following plan for unification of QT with GR [8]:

- Unification of all physical interactions in a single classical field model $\mathcal{M}$.

- Emergence of QT from $\mathcal{M}$.

Of course, $\mathcal{M}$ has to be local and to have nothing to do with nonlocal subquantum models of the Bohmian type.

One may immediately say that such $\mathcal{M}$ does not exist as the result of the experimental confirmation of a violation of the Bell inequality. However, this conclusion depends on treatments of possible ways of coupling a subquantum (ontic) model with QM (epistemic model), see [8] for discussion. In short, a violation of the Bell inequality closed only one special way for coupling of subquantum and quantum models. One can still treat QM as emergent from a causal classical-like model $\mathcal{M}$ [40].

For us, it is important that $\mathcal{M}$ can be a classical field model. One of such models was developed in the series of the works of the author and coauthors [76-83], so called prequantum classical statistical field theory (PCSFT), see monograph [40] for the detailed presentation. The idea behind this model and emergence of QM is very simple: in fact, quantum density operators are just the symbolic representations of covariance operators of classical random fields fluctuating at the time and space scales

21 See, e.g., Bohr's reply [74] to the EPR-paper [75]: "In this connection a viewpoint termed "complementarity" is explained from which quantum-mechanical description of physical phenomena would seem to fulfill, within its scope, all rational demands of completeness." 
which are essentially finer than the scales of quantum experimental physics. We are not able to monitor individual fluctuations; only correlations can be measured.

There is a kind of nonlocal element in PCSFT. However, this is not nonlocality of mystical action at a distance, but the presence of correlations in the common background field feeling the space-time (similar to the zero-point field). Its correlations contribute into the "quantum correlations." We repeat once again that correlations in such a background field are typical for, e.g., radio-engineering.

\section{What is to be Done?}

Now I would like to summarize the discussion with student, see Sect. 2, and my own reflections induced by this discussion, Sects. 4-6:

1. Both QM and QFT were just steps towards real QT which have not yet been constructed.

2. Nonrelativistic QM versus relativistic QFT.

3. QFT suffers of infinities. Ignorance of infinities under the shadow of regularization procedures cannot be considered as acceptable.

4. Infinities and singularities are foreign to real physical phenomena. A theory containing singularities cannot be considered as physically adequate, neither QFT nor Big Bang.

5. Since not QM, but QFT is considered as fundamental QT-model, the basic output of foundational studies in the QM framework, namely, the foundational output of violation of Bell's inequality - existence of action at a distance, has to be translated to the QFT-language.

6. The latter seems to be impossible, since there is no the QFT-based notion of entanglement, it is frame dependent.

7. Unification of QT with GR is a very risky adventure, because the real QT has not yet been created. Its initially created part in the form of QM suffers of the absence of relativistic invariance, it advanced part, QFT, suffers of infinities and problems with relativistic notion of entanglement.

Our analysis of the basic problems of QT (QM-QFT) and corresponding complications preventing unification of QT and GR has not to lead to pessimistic conclusions. Following to one of the first Russian revolutionaries, Nikolay Chernyshevsky [84], we can ask: "What is to be done?"

1. To create QFT which will be free of infinities (may be by following Dirac [42]).

2. If elimination of infinities were possible only through violation of relativistic invariance of QFT, we have to estimate consequences of such a change for the physics in whole.

3. We may hope that in such a new QT based on divergence free QFT action at a distance would get a new interpretation.

4. The use of real (and, hence, complex) numbers and Hilbert space are very strong mathematical constraints. Physical justification of their use can be questioned. 
5. Hilbert state space is so foreign to gravity, curved spaces and cosmology (see 't Hooft [53]) that it seems to be natural to try to explore non-Hilbertian models in the spirit of P. Dirac to proceed to unification of QT with GR.

6. In models based on non-Archimedean space the problem of (non-)locality is represented in the new way. There is not so much difference between classical and quantum models, they both are nonlocal from the viewpoint of the real metric.

7. By rejecting the impossibility to treat QM-QFT as emergent from classical-like causal theories we open new way to merge QT and GR through unification at the level of classical field theory. This way can be called "Einstein's dream way", see [39],[38]. Our PCSFT-model can serve as one of possible candidates for such a unification.

We finish our paper by the famous citation of P. Dirac [85], p. 85:

"It seems clear that the present quantum mechanics is not in its final form. Some further changes will be needed, just about as drastic as the changes made in passing from Bohr's orbit theory to quantum mechanics. Some day a new quantum mechanics, a relativistic one, will be discovered, in which we will not have these infinities occurring at all. It might very well be that the new quantum mechanics will have determinism in the way that Einstein wanted." 22

Acknowledgements This paper was supported by the grant of Faculty of Technology of Linnaeus University "Mathematical modeling of complex hierarchic systems" and the Grant of the EU-network QUARTZ.

Open Access This article is distributed under the terms of the Creative Commons Attribution 4.0 International License (http://creativecommons.org/licenses/by/4.0/), which permits unrestricted use, distribution, and reproduction in any medium, provided you give appropriate credit to the original author(s) and the source, provide a link to the Creative Commons license, and indicate if changes were made.

\section{Appendix}

1. QM and Special Relativity Theory (SRT) Relativistic Quantum Field Theory is a mathematical scheme to describe the sub-atomic particles and forces. The basic starting point is that the axioms of Special Relativity on the one hand and those of Quantum Mechanics on the other, should be combined into one theory. ... Since the energies available in sub-atomic interactions are comparable to, and often larger than, the rest mass energy $m c^{2}$ of these particles, they often travel with velocities close to that of light, $c$, and so relativistic effects will also be important. Thus, in the first half of the twentieth century, the question was asked: How should one reconcile Quantum Mechanics with Einstein's theory of Special Relativity? Quantum Field Theory is the answer to this question”. (See “t Hooft [88].)

"One can say that QFT results from the successful reconciliation of QM and SRT. ... There is also a manifest contradiction between QM and SRT on the level of the dynamics. The Schrdinger equation, i.e. the fundamental law for the temporal evolution of the quantum mechanical state function, cannot possibly obey

\footnotetext{
22 And, as we emphasized in this note, such a divergence free and relativistic quantum theory would be able to merge peacefully with general relativity.
} 
the relativistic requirement that all physical laws of nature be invariant under Lorentz transformations. The Klein-Gordon and Dirac equations, resulting from the search for relativistic analogues of the Schrdinger equation in the 1920s, do respect the requirement of Lorentz invariance. Nevertheless, ultimately they are not satisfactory because they do not permit a description of fields in a principled quantum-mechanical way." (See Kuhlmann [86].)

2. QFT-Foundational Studies As was rightly pointed out by one the reviewers of this paper: "Apart from the problem of the unification or compatibility with $G R, \ldots Q F T$ is far from complete or unified. Two main parts of the Standard Model of the elementary particle physics, the electroweak theory, unifying quantum electrodynamics (QED) and the theory of weak interaction, and quantum chromodynamics (QCD), which handles the strong interactions, are fully compatible but not really unified (there is no single established symmetry group). There are also a variety of foundational approaches to QFT in the first place”. Some of these approaches are discussed in Kuhlmanns review article [86]. And the main stream of QFT-foundational studies is directed towards the resolution of the special QFT interpretational problems, e.g., particle versus field interpretations of QFT. However, as was mentioned in introduction, a few authors contributed to quantum information related problems of the QFT-foundations, see, e.g., D' Ariano [11], Bisio, D' Ariano and Perinotti [12], and Plotnitsky [13-15].

3. QFT-Divergences It was again rightly pointed out by the same reviewer: "Certainly, the question of divergences and renormalization has been discussed and the presence of divergences has seen as problems throughout the history of QFT. Such important (more) recent developments as renormalization group and effective QFTs were in part responding to these problems". As a consequence, nowadays practically nobody claims that QFT should be rejected for manipulating with nonphysical (infinite) entities, cf. with 't Hooft's historical account of development of QFT [87], p. 3: "Before 1970, the particle physics community was (unequally) divided concerning the relevance of quantized fields for the understanding of subatomic particles and their interactions. On hindsight, one can see clearly why the experts were negative about this approach. Foremost was the general feeling that this theory was ugly, requiring various fixes to cover up its internal mathematical inconsistencies". Then, see [87], pp. 3-21, he continues to describe further development of QFT as successful (although difficult) resolution of all these inconsistencies. ${ }^{23}$ Consequently nowadays there is no this general feeling that QFT is an ugly and inconsistent theory (cf. with Dirac [42], [85] and with the above citation of ' $t$ Hooft).

\section{References}

1. Bell, J.S.: On the Einstein-Podolski-Rosen paradox. Physics 1, 195 (1964)

\footnotetext{
23 This great project was based on the contributions of Kramers, Schwinger, Dyson, Tomonaga, Feynman, Landau, Gell-Mann and Low, ..., De Witt, Faddeev, Popov, Feynman and Stanley Mandelstam, Slavnov, Taylor, ...., Englert, Brout, Higgs and Weinberg,...., Veltman, and, 't Hooft by himself, see [87], pp. 22-25, for the corresponding references.
} 
2. Bell, J.S.: Speakable and Unspeakable in Quantum Mechanics. Cambridge Univ. Press, Cambridge (1987)

3. Hensen, B., et al.: Experimental loophole-free violation of a Bell inequality using entangled electron spins separated by $1.3 \mathrm{~km}$. Nature 526, 682 (2015)

4. Giustina, M., et al.: A significant-loophole-free test of Bell's theorem with entangled photons. Phys. Rev. Lett. 115, 250401 (2015)

5. Shalm, L.K., et al.: A strong loophole-free test of local realism. Phys. Rev. Lett. 115, 250402 (2015)

6. Aspect, A.: Closing the door on Einstein and Bohr's quantum debate. Physics 8, 123 (2015)

7. Wiseman, H.: Quantum physics: death by experiment for local realism. Nature 526, 649650 (2015)

8. Khrennikov, A.: After Bell. Fortschritte der Physik- Prog. Phys. (2016). 10.1002/prop.201600044/ epdf

9. Kupczynski, M.: Can Einstein with Bohr debate on quantum mechanics be closed? arXiv:1603.00266 (quant-ph)

10. Stachel, J., Bradonjic, K.: Quantum gravity: meaning and measurement. Stud. Hist. Philos. Sci. Part B 46, 209-216 (2014)

11. D' Ariano, G. M.: Physics as quantum information processing: quantum fields as quantum automata. In: Foundations of Probability and Physics_6, M. D' Ariano , Sh.-M. Fei , E. Haven, B. Hiesmayr , G. Jaeger, A. Khrennikov and J.-A. Larsson (eds.), AIP Conf. Proc., 1424, pp. 371-386 (2012)

12. Bisio, A., D' Ariano, G.M., Perinotti, P.: Quantum walks, deformed relativity and Hopf algebra symmetries. Theme issue "Quantum foundations: information approach" D' Ariano, G.M. and Khrennikov, A. (eds.).' Phil. Trans. R. Soc. A 374, 20150232 (2016)

13. Plotnitsky, A.: A matter of principle: the principles of quantum theory, Dirac's equation, and quantum information. Found. Phys. 45, 12221268 (2015)

14. Plotnitsky, A.: The Principles of Quantum Theory, From Planck's Quanta to the Higgs Boson: The Nature of Quantum Reality and the Spirit of Copenhagen. Springer, Berlin (2016)

15. Plotnitsky A.: The future (and past) of quantum theory after the Higgs boson: a quantum-informational viewpoint. Theme issue "Quantum foundations: information approach” D' Ariano, G.M. and Khrennikov, A. (eds.). Phil. Trans. R. Soc. A 374, 20150239 (2016)

16. Teller, P.: An Interpretative Introduction to Quantum Field Theory. Princeton Univ. Press, Princeton (1995)

17. Saunders, S., Brown, H.R. (eds.): The Philosophy of Vacuum. Clarendon Press, Oxford (1991)

18. Brown, H.R., Harre, R. (eds.): Philosophical Foundations of Quantum Field Theory. Oxford Univ. Press, Oxford (1988)

19. Ruetsche, L.: Interpreting Quantum Theories. Oxford Univ. Press, Oxford (2011)

20. Davies, E., Lewis, J.: An operational approach to quantum probability. Commun. Math. Phys. 17, 239-260 (1970)

21. Okamura, K., Ozawa, M.: Measurement theory in local quantum physics. J. Math. Phys. 57, 015209 (2016)

22. Busch, P., Lahti, P.J., Pellonpää, J.-P., Ylinen, K.: Quantum Measurement. Springer, Berlin (2016)

23. Jaeger, G.: Macroscopic realism and quantum measurement: measurements as a natural kind. Phys. Scripta T 163, 014017 (2014)

24. Schweber, A.S.: An Introduction to Relativistic Quantum Field Theory. Dover Publ INC, Mineola (1989)

25. Schwinger, J.: Particles, Sources, and Fields. Westview Press, Boulder (1988)

26. Bohr, N. and L. Rosenfeld, L.: On the question of the measurability of electromagnetic field quantities, (in J. A. Wheeler \& W. H. Zurek, eds., Quantum theory and measurement, pp. 479 522. Princeton University Press, Princeton, NJ (1983)

27. Bohr, N. and L. Rosenfeld, L.: Field and charge measurements in quantum electrodynamics (in J. A. Wheeler \& W. H. Zurek (eds., Quantum theory and measurement, pp. 523534, Princeton University Press, Princeton (1983)

28. D'Agostino, S.: The Bild conception of physical theory: Helmholtz, Hertz, and Schrödinger. Phys. Perspect. 6, 372-389 (2004)

29. D'Agostino, S.: Continuity and completeness in physical theory: Schrödinger's return to the wave interpretation of quantum mechanics in the 1950's. In: Bitbol, M. and O. Darrigol (eds.) E. Schrödinger: Philosophy and the Birth of Quantum Mechanics, pp. 339-360, Editions Frontieres, Gif-sur-Yvette (1992) 
30. Atmanspacher, H., Bishop, R. C. and Amann, A.: Extrinsic and intrinsic irreversibility in probabilistic dynamical laws, , Foundations of Probability and Physics, pp. 50-70, World Scientific, Singapore(2001)

31. Atmanspacher, H.: Determinism is ontic, determinability is epistemic. In: Atmanspacher, H., Bishop, R.C. (eds.) Between Chance and Choice: Interdisciplinary Perspectives on Determinism, pp. 49-74. Imprint Academic, Thorverton UK (2002)

32. Atmanspacher, H. and Primas, H.: Epistemic and ontic quantum realities. In: Foundations of Probability and Physics-3, pp. 49-62. (AIP Conf. Proc.) 750, Melville, NY (2005)

33. Jaeger, G.: Entanglement, Information, and the Interpretation of Quantum Mechanics (The Frontiers Collection). Springer, Berlin (2009)

34. Jaeger, G.: Quantum Objects: Non-local Correlation, Causality and Objective Indefiniteness in the Quantum World (Ser. Fundamental Theories of Physics). Springer, Berlin(2014)

35. Jaeger, G.: Grounding the randomness of quantum measurement. Phil. Trans. Royal Soc. Lond A 374, $20150238(2016)$

36. von Neumann, J.: Mathematical Foundations of Quantum Mechanics. Princeton Univ. Press, Princeton (1955)

37. Dirac, P.A.M.: The Principles of Quantum Mechanics. Clarendon Press, Oxford (1995)

38. Khrennikov, A.: Einstein's dream. The nature of light: What are photons? Roychoudhuri, C., Kracklauer, A.F., Creath, K. (eds.). Proc. SPIE, 6664, 666409-1 - 666409-9 (2007)

39. Einstein, A., Infeld, L.: Evolution of Physics: the Growth of Ideas from Early Concepts to Relativity and Quanta. Simon and Schuster, New-York (1961)

40. Khrennikov, A.: Beyond Quantum. Pan Stanford Publ, Singapore (2014)

41. Riordan, M.: Tackling infinity. (Review about the book of F. Close, The infinity puzzle: Quantum Field Theory and the Hunt for an Orderly Universe, Basic Books, 2011.) American Scientist, 2016, http:// www.americanscientist.org/bookshelf/pub/tackling-infinity

42. Dirac, P.A.M.: Lectures on Quantum Field Theory. New York; Academic Press, New York, Belfer Graduate School of Science, Yeshiva University (1966)

43. Gurzadyan, V.G. and Penrose, R.: Concentric circles in WMAP data may provide evidence of violent pre-Big-Bang activity. arXiv:1011.3706 (astro-ph.CO)

44. Efimov, G.V.: Nonlocal quantum field theory, nonlinear interaction lagrangians, and the convergence of the perturbation-theory series. Theor. Math. Phys. 2, 217223 (1970)

45. Pais, A.: Inward Bound: Of Matter and Forces in the Physical World. Clarendon Press, Oxford (1986)

46. Dirac, P.A.M.: Lectures on Quantum Mechanics and Relativistic Field Theory. Martino Fine Books, Eastford (2012)

47. Smolyanov, O.G.: Infinite-dimensional pseudo-differential operators and Schrödinger quantization. Dokl. Akad. Nauk SSSR 263, 558-562 (1982)

48. Khrennikov, A.: Equations with infinite-dimensional pseudo-differential operators. Dokl. Acad. Nauk USSR 267, 1313-1318 (1982)

49. Khrennikov, A.: Second quantization and pseudo-differential operators. Theor. Math. Phys. 66, 339349 (1986)

50. Khrennikov, A.: Infinite-dimensional pseudo-differential operators. Izvestia Akad. Nauk USSR, ser.Math. 51, N. 6, 46-68 (1987); English translation: Math. USSR Izv. 31, pp 575-597 (1988)

51. Smolyanov, O.G., Khrennikov, AYu.: An algebra of infinite-dimensional pseudodifferential operators. Dokl. Akad. Nauk SSSR 292, 1310-1314 (1987)

52. A. Khrennikov, Infinite-Dimensional equation of Liouville. Mat. Sbornik, 183, 20-44 (1992); English translation: Russ. Acad. Sci. Sbornik Mathematics 75, 17 (1993)

53. 't Hooft, G.: The nature of quantum mechanics. Conference the future of physics, Santa Barbara, 2004. http://online.itp.ucsb.edu/online/kitp25/thooft/oh/01.html

54. Khrennikov, A.: Non-Archimedean analysis: quantum paradoxes, dynamical systems and biological models. Kluwer, Dordreht (1997)

55. Volovich, I.: Number theory as the ultimate physical theory, P-Adic Numbers, Ultrametric Anal. Appl., 2 (2010), 7787; preprint 1987

56. Volovich, V.: p-Adic string. Class. Quantum Gravity 4(4), L83 (1987)

57. Volovich, I.V.: p-Adic space-time and string theory. Theoret. Math. Phys. 71(3), 574576 (1987)

58. Vladimirov, V.S., Volovich, I.V., Zelenov, E.I.: p-adic analysis and mathematical physics, Series on Soviet and East European Mathematics, 1. WSP, River Edge, NJ (1994) 
59. Khrennikov, A.: p-adic valued distributions and their applications to the mathematical physics. Kluwer, Dordreht (1994)

60. Dragovich, B., Khrennikov, A., Kozyrev, S.V., Volovich, I.V.: On $p$-adic mathematical physics. $p$-Adic Numb. Ultramet. Anal. Appl. 1, 1-17 (2009). http://arxiv.org/pdf/0904.4205.pdf

61. Khrennikov, A.Y.: Mathematical Methods of non-Archimedean physics. Russ. Math. Surv. 45, 87 (1990)

62. Khrennikov, A.: p-adic quantum-classical analogue of the Heisenberg uncertainty relations. Nuovo Cimento 112, 555-560 (1996)

63. Albeverio, S., Khrennikov, A., Cianci, R.: On the spectrum of the $p$-adic position operator. J. Phys. A Math. Gen. 30, 881-889 (1997)

64. Albeverio, S., Bayod, J.M., Perez-Garcia, C., Cianci, R., Khrennikov, A.: Non-Archimedean analogues of orthogonal and symmetric operators and p-adic quantization. Acta Appl. Math. 57, 205-207 (1999)

65. Khrennikov, A.: An extension of the frequency approach of R. von Mises and the axiomatic approach of N.A. Kolmogorov to the $p$-adic theory of probability. Theor. Probab. Appl. 463, 458-463 (1995)

66. Khrennikov, A.: The Einstein-Podolsky-Rosen paradox and the $p$-adic probability theory. Dokl. Math. 54, 790-795 (1996)

67. Cianci, R., Khrennikov, A.: Can $p$-adic numbers be useful to regularize divergent expectation values of quantum observables? Int. J. Theor. Phys. 33(6), 1217-1228 (1994)

68. Cianci, R., Khrennikov, A.: p-adic numbers and the renormalization of eigenfunctions in quantum mechanics. Phys. Lett. B 328, 109-112 (1994)

69. Robinson, A.: Non-standard Analysis. North-Holland Publishing Co., Amsterdam (1966)

70. Kelemen, P.J., Robinson, A.: The non standard $\lambda: \phi_{2}^{4}(x)$ : model: I. The technique of non standard analysis in theoretical physics. J. Math. Phys. 13, 1870-1874 (1972)

71. Kelemen, P.J., Robinson, A.: The standard model from a non standard point of view. J. Math. Phys. 13, 1875-1878 (1972)

72. Albeverio, S., Fenstad, J.E., Hoegh-Krohn, R.: Singular perturbations and nonstandard analysis. Trans. Am. Math. Soc. 252, 275-295 (1979)

73. S. Albeverio, J. E. Fenstad, R. Hoegh-Krohn,. Lindstrom, Nonstandard Methods in Stochastic Analysis and Mathematical Physics. the Academic Press, Inc. Orlando, Florida, (1986)

74. Bohr, N.: Can quantum-mechanical description of physical reality be considered complete? Phys. Rev. 48, 696-702 (1935)

75. Einstein, A., Podolsky, B., Rosen, N.: Can quantum-mechanical description of physical reality be considered complete? Phys. Rev. 47, 777-780 (1935)

76. Khrennikov, A.: A pre-quantum classical statistical model with infinite-dimensional phase space. J. Phys. A 38, 9051 (2005)

77. Khrennikov, A.: Prequantum classical statistical field theory: complex representation, HamiltonSchrödinger equation, and interpretation of stationary states. Found. Phys. Lett. 18, 637 (2006)

78. Khrennikov, A.: Entanglement's dynamics from classical stochastic process. EPL 88, 40005 (2009)

79. Khrennikov, A., Ohya, M., Watanabe, N.: Classical signal model for quantum channels. J. Russ. Laser Res. 31, 462 (2010)

80. Khrennikov, A., Ohya, M., Watanabe, N.: Quantum probability from classical signal theory. Int. J. Quantum Inf. 9(1), 281-292 (2011)

81. Khrennikov, A.: Subquantum nonlocal correlations induced by the background random field. Phys. Scripta 84, 045014 (2011)

82. Khrennikov, A., Nilsson, B., Nordebo, S.: On an experimental test of prequantum theory of classical random fields : an estimate from above of the coefficient of second-order coherence. Int. J. Quantum Inf. 10, 1241014 (2012)

83. Khrennikov, A., Nilsson, B., Nordebo, S.: Quantum rule for detection probability from Brownian motion in the space of classical fields. Theor. Math. Phys. 174, 298-306 (2013)

84. Chernyshevsky, N.: Chto delatj? (In Russian), St. Petersburg, 1883. English Edition: What is to be done? Facsimile Publisher, 2016 (reprinted from the first English edition, 1886)

85. Dirac, P.: The early years of relativity. In: Holton, G.J., Elkana, Y. (eds.) Albert Einstein : Historical and Cultural Perspectives : the Centennial Symposium in Jerusalem (1979), pp. 79-90. Princeton Press, Princeton (1982)

86. Kuhlmann, M.: Quantum field theory, in Zalta, E. N. (ed.), The Stanford encyclopedia of philosophy, Summer 2015 ed., http://plato.stanford.edu/archives/sum2015/entries/quantum-field-theory/ 
87. 't Hooft, G.: The evolution of quantum field theory: from QED to grand unification. arXiv:1503.05007 (hep-th)

88. 't Hooft, G.: The conceptual basis of quantum field theory. http://www.staff.science.uu.nl/ hooft101/ lectures/basisqft.pdf 